Check for updates

Cite this: RSC Adv., 2017, 7, 32749

Received 20th March 2017

Accepted 22nd June 2017

DOI: 10.1039/c7ra03271d

rsc.li/rsc-advances

\title{
MEMS-based column coated with reduced graphene oxide as stationary phase for gas chromatography
}

\author{
Huan Yuan, $\mathbb{D}^{a}$ Xiaosong Du, ${ }^{\star a}$ Huiling Tai, ${ }^{a}$ Xiao Yang $^{a}$ and Ming $\mathrm{Xu}^{\text {*b }}$
}

This work presents the investigation of reduced graphene oxide (RGO) as a new type of stationary phase for gas chromatographic separation on a microcolumn which is fabricated on a silicon wafer by the application of microelectromechanical system (MEMS) techniques. A ZnO film synthesized by a sol-gel method is utilized as a supporting material between RGO and the column wall for inwall roughening to form a stable RGO stationary phase and increase gas-stationary phase interaction. The RGO/ZnO grafted on the column wall was characterized by SEM and FT-IR spectra. The experimental results demonstrate that good separations for mixtures of a wide range of organic compounds including alkanes and esters are achieved by the microcolumn coated with bilayer film. And the maximum column efficiency of 11363 theoretical plates per meter for $n$-dodecane is obtained, which is higher than any previously reported values for chromatography columns coated with graphene and their analogs to the best of our knowledge. However, the RGO stationary phase shows obvious peak tailings for polar alcohols, which mainly originate from its specific $\pi-\pi$ stacking interaction as well as the hydrophobic effect between analytes and the $\pi$-electron on the high surface area of reduced graphene oxide. This work greatly favors RGO coated chip columns for gas chromatographic separation in achieving micro-total analytical systems.

\section{Introduction}

Graphene (G), a carbon monolayer packed into a 2D honeycomb lattice, is a basic building block for graphitic materials of all other dimensionalities. ${ }^{1}$ Since its discovery by Novoselov $e t ~ a l .{ }^{2}$ in 2004, intense research efforts have been focused on its wide range of potential applications and very promising array of features ${ }^{3}$ with respect to other structurally different forms of carbon. ${ }^{4}$ Graphene provides many excellent properties, ${ }^{5}$ such as large surface area $\left(2630 \mathrm{~m}^{2} \mathrm{~g}^{-1}\right),{ }^{6}$ high mechanical strength $(\sim 1000 \mathrm{GPa})^{7}$ and high electron mobility rate $\left(200000 \mathrm{~cm}^{2} \mathrm{~V}^{-1}\right.$ $\left.\mathrm{s}^{-1}\right) .{ }^{8}$ Thus, graphene has emerged as a promising material for energy, ${ }^{9}$ electronics,${ }^{10}$ biomedicine, ${ }^{11}$ composite science ${ }^{12}$ and so on. In the field of analytical chemistry, graphene as well as its analogs such as graphene oxide (GO) also shows great potential applications. ${ }^{13-16}$ As a new adsorption material, Liu $^{13}$ demonstrated that analytes such as chlorophenols can be effectively extracted by graphene in solid-phase extraction. Chen ${ }^{16}$ evaluated a graphene based solid-phase microextraction (SPME) fiber

\footnotetext{
${ }^{a}$ State Key Laboratory of Electronic Thin Films and Integrated Devices, University of Electronic Science and Technology of China, Chengdu 610054, China. E-mail: xsdu@uestc.edu.cn

${ }^{b}$ Key Laboratory of Information Materials of Sichuan Province, School of Electrical and Information Engineering, Southwest University for Nationalities, Chengdu 610041, China.E-mail: hsuming_2001@aliyun.com
}

through extraction of six pyrethroid pesticides. On the other hand, considering its high surface area, graphene-like materials have drawn tremendous attention as stationary phase for a variety of chromatographic set-ups. ${ }^{17-23}$ Among them, graphene oxide and graphene have been intensely studied. Zhang ${ }^{17}$ introduced GO sheets onto amino silica microspheres via covalent coupling into a high performance liquid chromatography (HPLC) using some coupling agents, and subsequently graphene (G) functionalized HPLC stationary phase was prepared through hydrazine reduction of GO bonded silica composite. Effective separations of the tested neutral and polar compounds on both columns were achieved. $\mathrm{Liu}^{18}$ reported the application of graphene oxide and reduced graphene oxide (RGO) sheet as novel stationary phases for open-tubular capillary electro chromatography (OTCEC) based on electrostatic assembly. Efficient separations of all the analytes, including three acid nitrophenol isomers, three basic nitroaniline isomers, and four neutral polycyclic aromatic hydrocarbon, were achieved. Feng ${ }^{19}$ argued the GO nanosheets as gas chromatographic (GC) stationary phase for achieving good separation for H-bonding analytes with good peak shapes. Fan ${ }^{20}$ investigated the applicability of graphene as high temperature GC stationary phase to separate a mixture containing aromatics and $n$-alkanes, in which graphene capillary column was heated to $280{ }^{\circ} \mathrm{C}$ thanks to its high thermal stability. However, graphene as well as its analogs as stationary phase in LC and CEC 
exhibited severe peak tailings and poor resolution for some aromatics such as naphthalenes, biphenyls, alkylbenzenes, phthalate acid esters and nitroanilines. ${ }^{21,22}$ The similar results of low chromatographic peak symmetry and significant peak tailing were also found for most compounds especially for alcohols when using GO as stationary phase in a gas chromatography. ${ }^{23}$ The authors deduced that one possible reason for those peaks may be due to the presence of rich functional groups and the $\mathrm{sp}^{2}$-conjugated bond in the carbon lattice of GO.

As is well known, pure graphene is intrinsically rather inert, ${ }^{24}$ while graphene oxide bears a giant of carboxyl, hydroxyl, and epoxide groups which provide GO sheets strongly interact with compounds via the formation of hydrophobicity, hydrogen bonding, and $\pi-\pi$ electrostatic stacking, which are considered as one possible reason for obvious peak tailing by $\mathrm{Qu} .^{23}$ Thus, according to the theory of a chromatographic column, too little or too much oxygen functional groups in graphene as well as its analogs isn't helpful for them to act as a stationary phase to achieve good separation. On the contrary, as a kind of chemically derived graphene, RGO still contains residual ( $\sim 8$ atomic per cent) oxygen that is $\mathrm{sp}^{3}$ bonded to approximately $20 \%$ of the carbon atoms. ${ }^{25}$ The dangling oxygen atoms act as binding sites for analytes ${ }^{\mathbf{2 6}}$ and provide oxygen functional groups for affinity. Thus, reduced graphene oxide is chosen as the stationary phase for gas chromatography in this paper. As a result of recent survey in the literature, there is only one paper ${ }^{17}$ regarding RGO as a new stationary phase in chromatographic separation, and the new phase was used in CEC application and no publication on RGO GC stationary phase is reported till now.

In this paper, instead of using traditional capillary GC columns, we utilized a MEMS-based column for this study, since the developing of micro GC devices and systems is our main research effort. ${ }^{27-29}$ The MEMS-based columns were fabricated on a silicon wafer by deep silicon etching technique where reduced graphene oxide was immobilized onto the column wall through sol-gel-derived $\mathrm{ZnO}$ particles as a supporting layer between the RGO film and the channel wall. The rough $\mathrm{ZnO}$ underlayer not only contained a high surface area for the bilayer film structure to increase gas-stationary phase interaction but also provided an alternative way to coat the RGO film from the as-purchased aqueous solution. RGO stationary phase was evaluated by GC separations of different types of analytes, including alkanes, esters and alcohols.

\section{Materials and methods}

\subsection{Fabrication of the MEMS-based column}

The MEMS-based column was designed as multi-capillary column (MCC, 0.5 m-long, $300 \mu \mathrm{m}$-deep, $30 \mu \mathrm{m}$-wide and eight capillaries) to enlarge the sample capacity, just as reported in our previous work. ${ }^{27}$ The fabrication of the microcolumn was based on a siliconon-glass process. First, a $3 \mu \mathrm{m}$ thick layer of aluminum film served as a mask for silicon etching was deposited on a type $\langle 100\rangle$ silicon wafer by electron beam evaporation. Second, photoresist of approximately $4 \mu \mathrm{m}$ was coated on the wafer and patterned on the aluminum film as a mask for aluminum etching. After the unprotected parts of the aluminum film was etched away by an

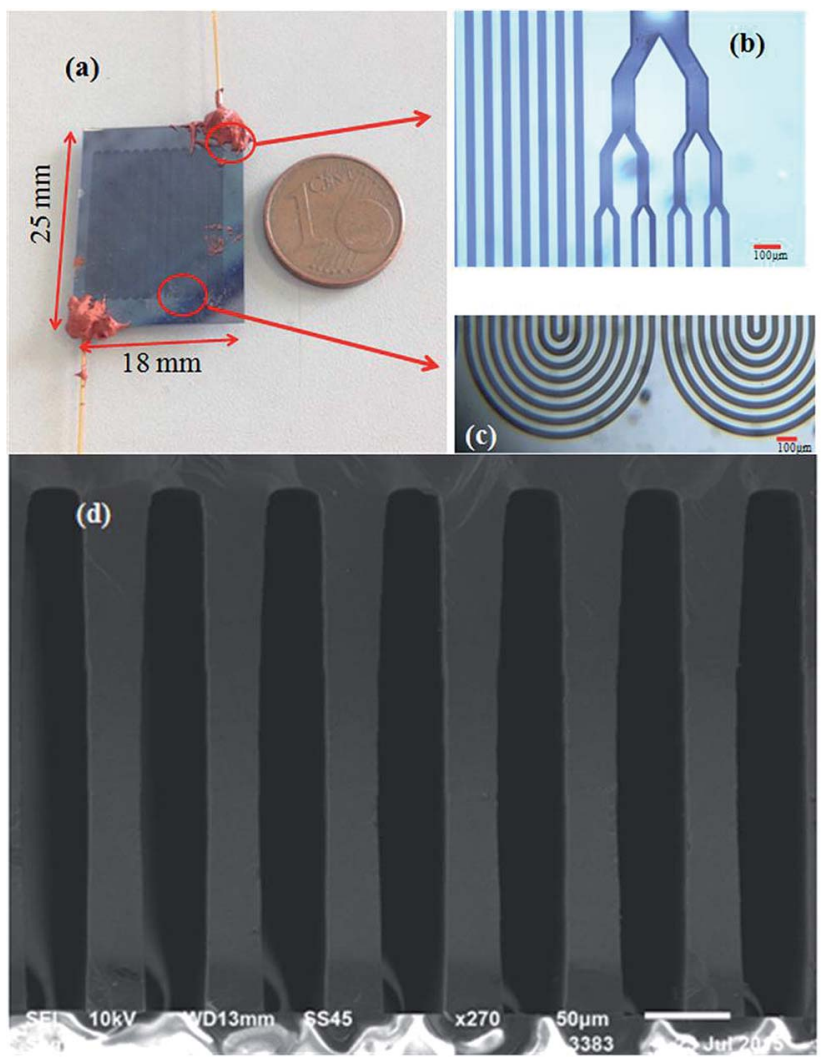

Fig. 1 The final size of the silicon wafer chip (a), the optical microscopy images of the inlet (b), the $90^{\circ}$ turning of the channel (c), and the SEM cross-sectional image of the MEMS-based eight-capillary column (d).

etchant, the wafer was etched to a depth of $300 \mu \mathrm{m}$ using Deep Reactive Ion Etching (DRIE), which was performed by introducing two gases (sulfur hexafluoride, $\mathrm{SF}_{6}$, and octafluorocyclobutane, $\mathrm{C}_{4} \mathrm{~F}_{8}$ ) alternately into the chamber. The $\mathrm{SF}_{6}$ etched the silicon during the process while the $\mathrm{C}_{4} \mathrm{~F}_{8}$ cross-linked to a passivation layer that coated on the vertical sidewalls of the micro channels. Subsequently, the aluminum mask was stripped off and the silicon wafer was anodically bonded to a Pyrex 7740 glass in a high electric field at $350{ }^{\circ} \mathrm{C}$, thus sealed all the channels. Finally, the sealed devices were cut from the wafer, whereby exposing the inlet and outlet ports of the columns at the side of the silicon wafer. After cutting from the wafer, the chips were connected to a traditional GC instrument for testing by $0.2 \mathrm{~m}$ long fused silica capillary bare tubing of $430 \mu \mathrm{m}$ in outer diameter (OD) and $320 \mu \mathrm{m}$ in inner diameter (ID). A photograph of the fabricated MEMS column is shown in Fig. 1(a), with a size of $25 \mathrm{~mm} \times 18 \mathrm{~mm}$. Fig. 1(b) and (c) are enlarged optical microscope images of the inlet/outlet and the $90^{\circ}$ turning of the serpentine channel, respectively. The crosssectional SEM image of Fig. 1(d) highlights the high aspect ratio $(10: 1)$ of the deep vertical multi-capillary channels.

\subsection{Stationary phase coating}

As is well known, the stationary phase coating is more challenging for MEMS columns, particularly for large number of narrow channels. Two main methods are used in coating 
procedures, that is, static and dynamic. We chose the former. Before coating the stationary phase, the multi-capillary column was flushed with $5 \mathrm{ml}$ octamethylcyclotetrasiloxane (D4) to modify the inner surface of the column through a covalent interaction between $\mathrm{Si}-\mathrm{OH}$ groups and $\mathrm{D} 4 .^{30}$ To ensure complete precondition, the column was placed in an oven at $400{ }^{\circ} \mathrm{C}$ for 90 minutes purging with $\mathrm{N}_{2}$.

RGO was used as the stationary phase for the MCC. RGO aqueous solution (0.43 wt\%, RGO: $96.41 \%$, C: $3.59 \%$, O: $1-10$ layers) was purchased from Chengdu Organic Chemicals Co. Ltd., Chinese Academy of Sciences. However, the as-purchased aqueous solution can't be directly coated on the bared silicon side walls since the viscosity of the aqueous solution was much low. The same situation can be also found for the GO and G disperse solutions. Thus, Qu et al. ${ }^{23}$ used coupling agents such as 3-aminopropyldiethoxymethyl silane to coat graphene oxide stationary phase. However, the applicability of this method to coat RGO is relatively low because RGO possesses much less active functional groups compared to GO, resulting in not enough carboxyl groups $(\mathrm{COOH})$ in RGO to bind with the amino groups $\left(\mathrm{NH}_{2}\right)$ in the coupling agent. Therefore, we utilized $\mathrm{ZnO}$ underlayer to facilitate the RGO coating. The $\mathrm{ZnO}$ underlayer can effectively roughen the inner wall of the channel, which increasing gas-stationary phase interaction and improving the adhesion of the stationary phase for a more stable stationary phase film. ZnO films were synthesized by sol-gel method, just as reported in our previous work, ${ }^{31}$ acting as a supporting underlayer material for inwall roughening. Zinc acetate dihydrate was dissolved into ethanol with ethanolamine $\left(\mathrm{C}_{2} \mathrm{H}_{7} \mathrm{NO}\right)$ as stabilizer. The solution was stirred at $60^{\circ} \mathrm{C}$ for 2 hours to yield a stable and homogeneous precursor solution, until the sol $\mathrm{pH}$ value reached 8 conditioned with glacial acetic acid or ammonia. The solution was aged for 72 hours at room temperature and then was flushed to the GC column. To ensure the generated $\mathrm{ZnO}$ particles to complete coarsening, the column was placed in an oven at $400{ }^{\circ} \mathrm{C}$ for 3 hours while purging with $\mathrm{N}_{2}$ as carrier gas. $5 \mathrm{ml}$ of the as-purchased RGO solution ( $0.43 \mathrm{wt} \%$ equals to $4.15 \mathrm{mg} \mathrm{ml}^{-1}$ ) was diluted to $1.97 \mathrm{mg} \mathrm{ml}^{-1}$ by adding about $5.5 \mathrm{ml}$ deionized water. The RGO aqueous solution was pressed into the microcolumn with a nitrogen tank. The whole column was filled with the solution of the stationary phase and was sealed on both ends. The microcolumn was aged for 24 hours at room temperature and then the solution was pushed. The temperature of the column was increased from $30^{\circ} \mathrm{C}$ at a rate of $2{ }^{\circ} \mathrm{C} \mathrm{min}{ }^{-1}$ until it reached $200{ }^{\circ} \mathrm{C}$. The column was kept at $200{ }^{\circ} \mathrm{C}$ and washed using $\mathrm{N}_{2}$ for 4 hours to form the uniform stationary phase film.

\subsection{Apparatus and equipment}

To characterize the column performance, all separation experiments were performed with an Agilent GC 6890 equipped with a flame ionization detector (FID). The inlet temperature was set to $270{ }^{\circ} \mathrm{C}$ with an appropriate split ratio. Pure nitrogen was used as carrier gas, and the mixture of the analytes were injected by an external sample injector. The carrier gas velocity was controlled by the Agilent GC 6890 system.

\section{Results and discussions}

\subsection{The stationary phase film}

The schematic view of RGO thin film directly deposited onto the channel wall is illustrated in Fig. 2(a). It emphasizes that few gaps exist between the RGO film and the channel wall. However, in the case of formation of a supporting material, $\mathrm{ZnO}$, the most part of RGO is supported by the ZnO particles, and only a small fraction of RGO is in touch with the channel wall through the cracks between $\mathrm{ZnO}$ granules as depicted in Fig. 2(b). It could be concluded that more gaps would exist between the RGO film and the rough $\mathrm{ZnO}$ film in the bilayer film structure. The more adsorption sites exist in the bilayer film structure and more analyte molecules can access the adsorption sites from all directions because the analyte can diffuse not only from the surface but also from the sides through gaps between RGO and ZnO granules. ${ }^{32}$ Fig. 3(a) displays SEM image of a bare MEMSbased column. When the channel wall is coated by a $\mathrm{ZnO}$ underlayer, the SEM surface morphology of the ZnO film is shown in Fig. 3(f). It can be seen undoubtedly that the sol-gelderived $\mathrm{ZnO}$ film is composed of well dispersed nanoparticles with diameters between 30 and $40 \mathrm{~nm}$, resulting in a rough surface. Subsequently, when the RGO film is deposited on the $\mathrm{ZnO}$ underlayer, the bilayer film coated column is shown in Fig. 3(b) and (c). It is evidently that the immobilization of RGO nanosheets on the column inner wall is realized thanks to the rough surface provided by the $\mathrm{ZnO}$ underlayer. The crosssectional SEM images of the bilayer stationary phase coating are shown with increasing magnification in Fig. 3(d) and (e), we observe that the RGO film on the $\mathrm{ZnO}$ granules is evenly distributed and the thickness of the RGO film is the order of several tens of nanometers. The bilayer film would be in favor of increasing gas-stationary phase interaction than the counterpart using only RGO, resulting in higher separation efficiency.

The formation of the bilayer film was further supported by FT-IR spectra as shown in Fig. 4. According to Mattevi's argument, ${ }^{33} \mathrm{RGO}$, after reduction of GO, were reduced significantly in the oxygen containing groups, suggesting a considerable deoxygenation. Thus, the band at about $1730 \mathrm{~cm}^{-1}(\mathrm{C}=\mathrm{O}$ stretching vibration of the carboxyl group) is not obviously observed on the FT-IR spectrum..$^{34}$ While a weak peak at $\sim 1630$ $\mathrm{cm}^{-1}$ owing to aromatic $\mathrm{C}=\mathrm{C}^{35}$ can be found, indicating the existence of RGO. The peak at $\sim 430 \mathrm{~cm}^{-1}$ is the stretching mode

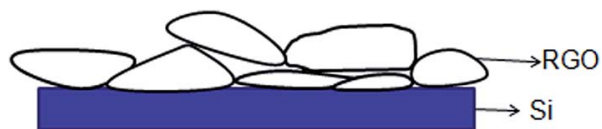

(a)

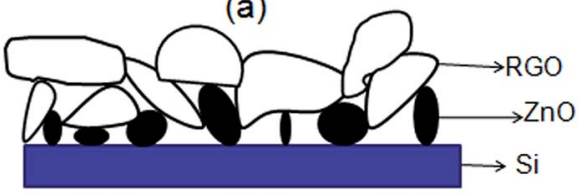

(b)

Fig. 2 Schematic diagram of single-layer stationary phase (a) and bilayer stationary phase (b). 

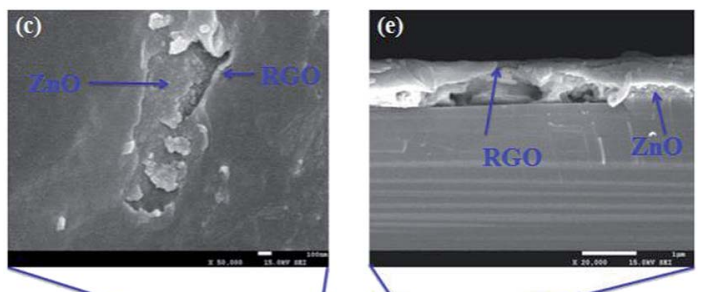

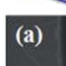

(a)
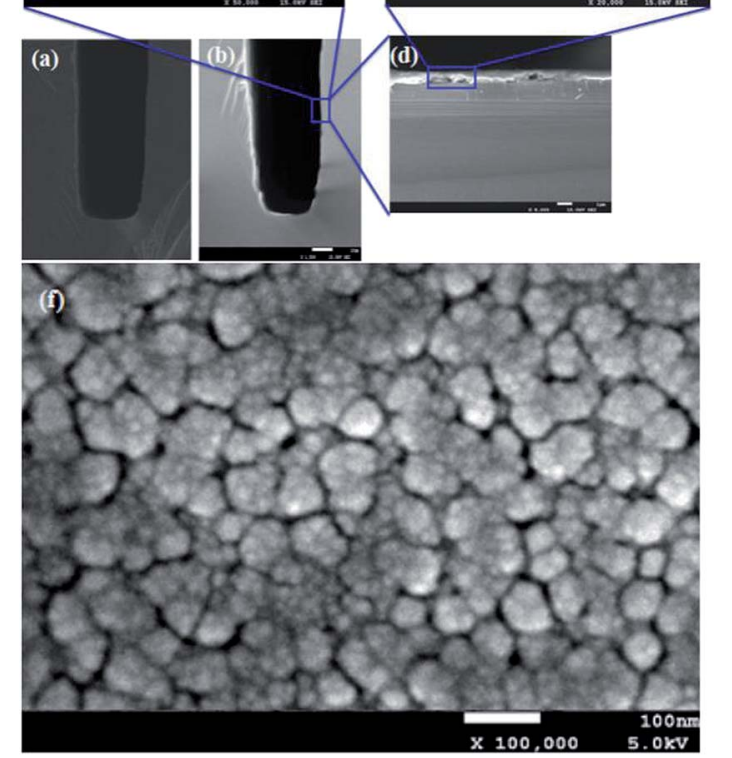

Fig. 3 SEM images of bare MEMS-based column channel (a), RGO/ ZnO bilayer coated channel (b), the surface of the bilayer coating (c), the cross section of bilayer coating with 8000 times (d), 20000 times (e), and surface morphology of $\mathrm{ZnO}$ underlayer ( $\mathrm{f}$ ).

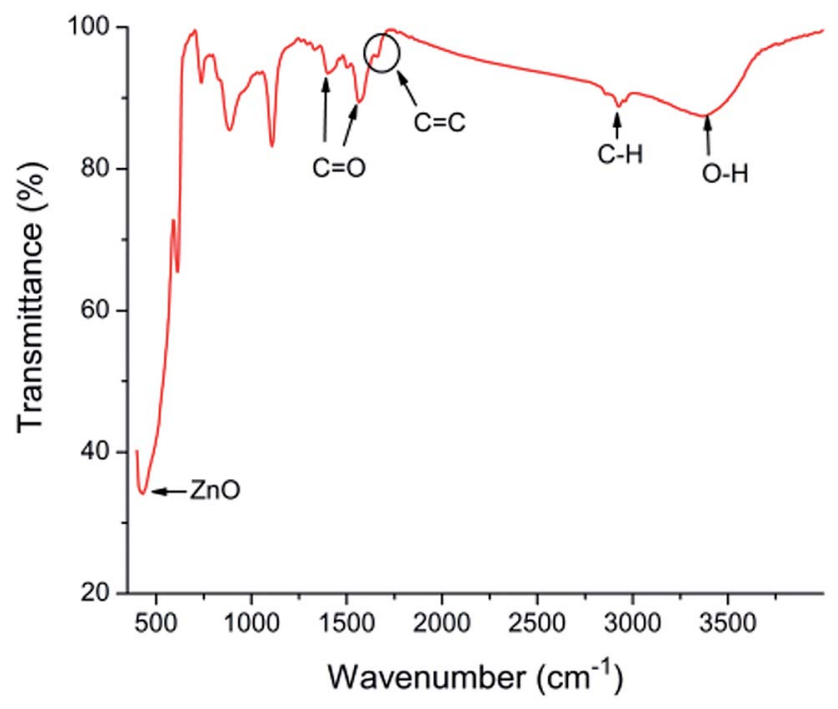

Fig. 4 FT-IR spectrum of the stationary phase.

of $\mathrm{ZnO}{ }^{36}$ However, the spectrum shows absorption bands at $\sim 2900, \sim 1400$ and $\sim 1600 \mathrm{~cm}^{-1}$, corresponding to the $\mathrm{C}-\mathrm{H}$ mode, asymmetric and symmetric $\mathrm{C}=\mathrm{O}$ stretching modes of zinc acetate, respectively, ${ }^{37}$ indicating the uncompletion of the sol-gel process. The unconverted reactant is also confirmed by the couple of vibrational bands at $3000-3500 \mathrm{~cm}^{-1}$ due to hydroxyl stretching vibrations of $\mathrm{COOH}$ and/or intercalated water. ${ }^{38}$

\subsection{The chromatographic separation}

We next turn to the analysis of separation experimental for the eight-capillary microcolumn. The column resolution and separation efficiency as two important figures of merit for the separation method were investigated in this paper. The column resolution is used to express the degree of separation between two adjacent peaks. A resolution of 1.5 denotes the two components are separated almost completely, with only $2 \%$ overlap. ${ }^{39}$ The separation efficiency is an important index to determine the GC column behaviour. This value can be described by the theoretical plate which is defined as a section of the column whereas the analyte is in equilibrium between the mobile and the stationary phases.

To test the separation performance, polar, weak polar and nonpolar component mixtures were injected into the column separately. $0.02 \mu \mathrm{l}$ of the mixture was injected with a split of $200: 1$. The inlet pressure was set at 3 psi during the experiment. The chip column was placed in the Agilent $6890 \mathrm{GC}$ oven and connected to the injector and the flame ionization detector (FID). $\mathrm{N}$-Alkanes $\left(\mathrm{C}_{5}-\mathrm{C}_{12}\right)$ were used as nonpolar analytes. The column was heated from $40{ }^{\circ} \mathrm{C}$ to $200{ }^{\circ} \mathrm{C}$ at a rate of $45^{\circ} \mathrm{C} \mathrm{min}{ }^{-1}$. The boiling points of the eight analytes range from $36{ }^{\circ} \mathrm{C}$ to $215^{\circ} \mathrm{C}$. As shown in Fig. 5(a), it is clear that all alkane components are completely separated except $\mathrm{C}_{5}$ and $\mathrm{C}_{6}$ on $\mathrm{RGO} / \mathrm{ZnO}$ coated microcolumn. This result indicates that the MCC can separate components with a wide range of boiling points within a short period of time $(<2.25$ minutes $)$. Thus, the relatively low resolution of 1.28 between $\mathrm{C}_{5}$ and $\mathrm{C}_{6}$ can be accepted rationally. It can be found that $\mathrm{N}$-alkanes are eluted in the order of increasing boiling points in Fig. 5(a). The number of theoretical plates per meter $(N)$ for these compounds varies between 84 and 6906.

Then the column was tested against weak polar molecules, wherein the column was heated from $40{ }^{\circ} \mathrm{C}$ to $200{ }^{\circ} \mathrm{C}$ at a rate of $30{ }^{\circ} \mathrm{C} \mathrm{min}^{-1}$. It can be seen in Fig. 5(b) that the three ester components are completely separated within 1.18 minutes. Although the chromatographic peaks present fairly good symmetry, peak broadening with respect to the nonpolar analytes can be evidently found. Meanwhile the plate numbers of all components are not high. One possible reason for these results is caused by the low distribution constant of each component in short column length. Nevertheless, the resolution is greater than 1.8, well above the criterion for baseline separation (a resolution of 1.5).

The microcolumn was also applied for the separation of polar analytes (four alcohol compounds). The initial temperature of GC column was set at $40^{\circ} \mathrm{C}$. Afterwards, the column was heated at a rate of $35{ }^{\circ} \mathrm{C} \mathrm{min}^{-1}$ until the temperature reached $180^{\circ} \mathrm{C}$. It can be seen from Fig. 5(c) that a complete separation is achieved with resolution factors greater than 2 . However, the peaks are broadened remarkably and show severe asymmetry.

The above findings demonstrate the unique separation performance of reduced graphene oxide stationary phase for 

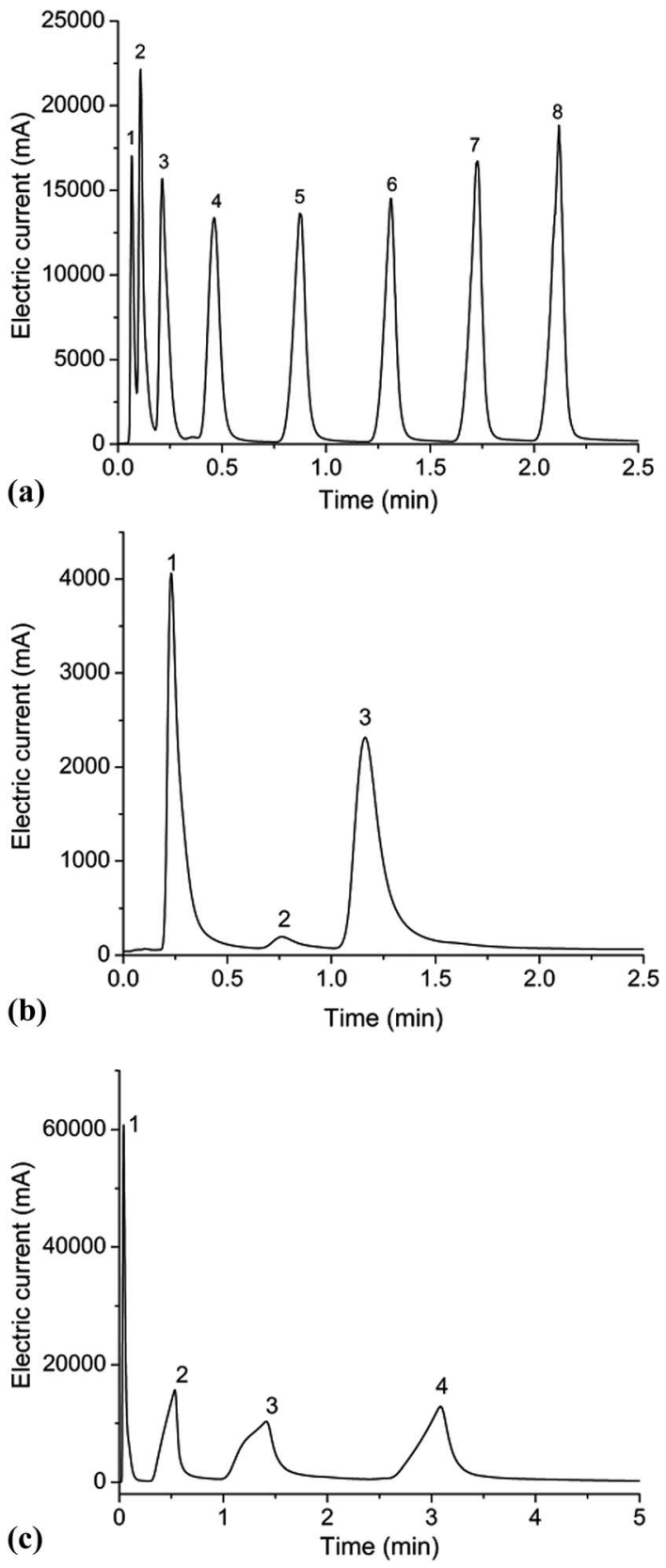

Fig. 5 Separations of the mixture of eight alkanes (a), three ester components (b), and four alcohols (c) by the RGO/ZnO column. The gas from component 1 to 8 in (a) is pentane, hexane, octane, nonane, decane, undecane and dodecane, respectively. The gas from component 1 to 3 in (b) is butyl acetate, ethyl lactate and ethyl heptanoate, respectively. The gas from component 1 to 4 in (c) is butyl alcohol, hexanol, octanol and lauryl alcohol, respectively.

a wide range of analytes, including alkanes, esters and alcohols. Being a new type of GC stationary phase, RGO phase achieves good separation for different kind of mixtures, especially for the $\mathrm{N}$-alkanes mixture, among them dodecane achieves an efficiency of 6906 theoretical plates per meter. Also, as evidenced above, RGO phase generally exhibits good separation for the esters and alcohols. However, it should be noted that in our experiments, obvious peak tailing is found for most compounds especially for alcohols. In addition, the alcohols show much more asymmetric broadening peaks. To understand these phenomena, the retention mechanism of RGO phase coated column must be addressed and one needs to know about the polarity of RGO phase. McReynolds constants for graphene and GO were determined on capillary columns by Qi's group. ${ }^{20}$ They pointed out that graphene exhibited weakly polar nature with McReynolds constants less than 100 . That's why our RGO coated column exhibits better separation performance for nonpolar and weak polar compounds such as alkanes and esters than polar alcohols according to like-dissolves-like rule. It is well-known that RGO has not much polar moieties, such as hydroxy, epoxy, and carboxy groups, ${ }^{40}$ in contrast, exhibiting a nonpolar and hydrophobic character induced by $\mathrm{sp}^{2}$-conjugated bond in the carbon of RGO. ${ }^{20}$ We think that RGO phase may involve multiple interactions in achieving its high resolving ability, including $\pi-\pi$ stacking, hydrogen-bonding and dispersion interactions which make RGO having a greater affinity for alcohols than alkanes and esters. This may be the reason of the more asymmetric broad peaks and higher peak tailing for alcohols than other analytes, which is in agreement with Qu's conclusion. ${ }^{23}$ These experimental results indicate that nonpolar interactions between the stationary phase and analytes contribute a lot for the separation on RGO coated columns.

The separation experiments were also conducted on a RGO stationary phase without $\mathrm{ZnO}$ underlayer. In the same conditions of preparation method and separation experimental parameters, the mixture of eight alkanes $\left(\mathrm{C}_{5}-\mathrm{C}_{12}\right)$ was injected into the RGO modified column. As shown in Fig. 6, the experimental result demonstrates very bad separation of the above alkanes. We believe the bad performance is due to the directly coated RGO stationary phase is unevenly distributed on the channel wall, since intrinsically GO nanosheets and its analogs tend to aggregate on a GC column. ${ }^{19}$

Meanwhile, a microcolumn only bonded with $\mathrm{ZnO}$ was tested by using identical separation conditions for comparison. As expected, ZnO film was not helpful for the separation and no

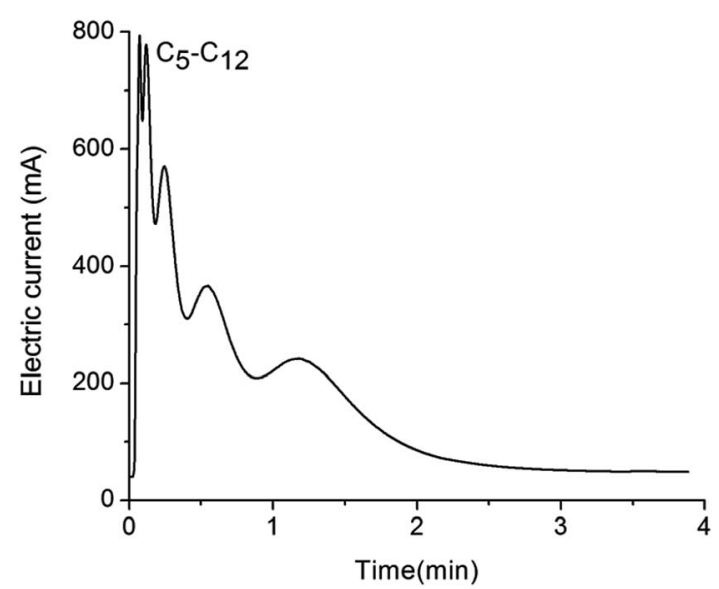

Fig. 6 Separation of the mixture of eight alkanes by RGO modified column without $\mathrm{ZnO}$ underlayer. 
separation was observed on this column. It confirmed that the separation of the $\mathrm{RGO} / \mathrm{ZnO}$ bilayer coating occurred through the interactions between RGO and the analytes.

\subsection{Column efficiency and repeatability}

Fig. 7 shows the Van Deemter plot for the RGO/ZnO coated column by isothermal determination of $n$-dodecane at $120^{\circ} \mathrm{C}$ at different carrier gas velocity. Dodecane, which is assumed to essentially be unretained, was injected into the eight-capillary column to obtain the corresponding HETP value. The

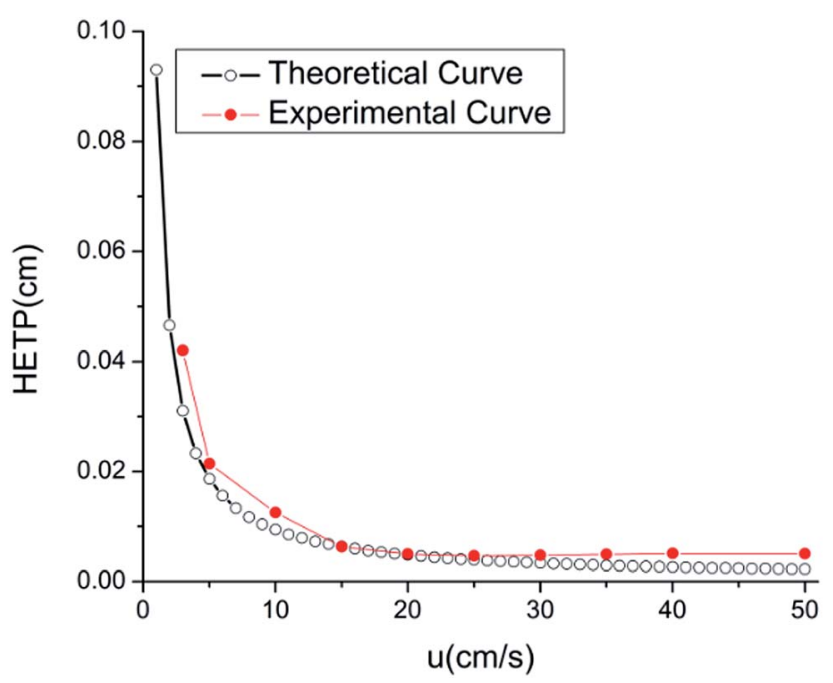

Fig. 7 Height equivalent to a theoretical plate versus average carrier gas velocity for the eight-capillary gas chromatography column.

Table 1 The repeatability datum of the RGO/ZnO column

Experiment number $(n=5)$

$\begin{array}{lllllll} & t_{\mathrm{R} 1} & t_{\mathrm{R} 2} & t_{\mathrm{R} 3} & t_{\mathrm{R} 4} & t_{\mathrm{R} 5} & \mathrm{RSD} \\ \text { Analyte } & (\mathrm{min}) & (\mathrm{min}) & (\mathrm{min}) & (\mathrm{min}) & (\mathrm{min}) & (\%)\end{array}$

(a) Alkanes

Pentane

Hexane

Heptane

Octane

Nonane

Decane

Undecane

Dodecane

(b) Esters

Butyl acetate

Ethyl lactate

Ethyl heptanoate

\subsection{0}

$$
0.761
$$

1.162

0.234

0.756

1.139

0.225
0.705

0.705
1.155

\subsection{0}

0.792

1.182

0.243

3.11

$1.179 \quad 4.44$

(c) Alcohols

Butyl alcohol

Hexanol

Octanol

\subsection{4}

\subsection{6}

1.287

0.054

0.472

0.048

0.055

0.572

$0.052 \quad 5.31$

Lauryl alcohol

1.232

$1.412 \quad 1.435$

$0.566 \quad 7.61$

$1.465 \quad 7.39$

$3.226 \quad 4.50$

\section{Acknowledgements}

This work was supported by the National High-Tech Research \& Development Program of China (Grant No. 2014AA06A510) and National Science Founds for Creative Research Groups of China (No. 61421002).

\section{References}

1 A. K. Geim and K. S. Novoselov, The rise of graphene, Nat. Mater., 2007, 6, 183-191.

2 K. S. Novoselov, A. K. Geim, S. V. Morozov, D. Jiang, Y. Zhang, S. V. Dubonos, I. V. Grigorieva and A. A. Firsov, Electric field effect in atomically thin carbon films, Science, 2004, 306, 666-669.

3 K. S. Novoselov, V. I. Fal'ko, L. Colombo, P. R. Gellert, M. G. Schwab and K. Kim, A roadmap for graphene, Nature, 2012, 490, 192-200.

4 Z. S. Wu, G. Zhou, L. C. Yin, W. Ren, F. Li and H. M. Cheng, Graphene/metal oxide composite electrode materials for energy storage, Nano Energy, 2012, 1, 107-131. 
5 V. Singh, D. Joung, L. Zhai, S. Das, S. I. Khondaker and S. Seal, Graphene based materials: past, present and future, Prog. Mater. Sci., 2011, 56, 1178-1271.

6 M. D. Stoller, S. Park, Y. Zhu, J. An and R. S. Ruoffet, Graphene-based ultracapacitors, Nano Lett., 2008, 8, 34983502.

7 C. Lee, X. Wei, J. W. Kysar and J. Hone, Measurement of the elastic properties and intrinsic strength of monolayer graphene, Science, 2008, 321, 385-388.

8 K. I. Bolotin, K. J. Sikes, Z. Jiang, M. Klima, G. Fudenberg, J. Hone, P. Kim and H. L. Stormer, Ultrahigh electron mobility in suspended graphene, Solid State Commun., 2008, 146, 351-355.

9 A. Varzi, S. Passerini, B. Scrosati and B. Scrosati, The role of graphene for electrochemical energy storage, Nat. Mater., 2015, 14, 271-279.

10 N. Weiss, H. Zhou, L. Liao, Y. Liu, S. Jiang, Y. Huang and X. F. Duan, Graphene: An emerging electronic material, Adv. Mater., 2012, 24, 5782-5825.

11 N. Mohanty and V. Berry, Graphene-based single-bacterium resolution biodevice and DNA transistor: interfacing graphene derivatives with nanoscale and microscale biocomponents, Nano Lett., 2008, 8, 4469-4476.

12 X. Y. Qi, K. Y. Pu, H. Li, X. Z. Zhou, S. X. Wu, Q. L. Fan, B. Liu, F. Boey, W. Huang and H. Zhang, Amphiphilic graphene composites, Angew. Chem., Int. Ed., 2010, 49, 9426-9429.

13 Q. Liu, J. Shi, L. Zeng, T. Wang, Y. Cai and G. Jiang, Evaluation of graphene as an advantageous adsorbent for solid-phase extraction with chlorophenols as model analytes, J. Chromatogr. A, 2011, 1218, 197-204.

14 Q. Ye, L. H. Liu, Z. B. Chen and L. M. Hong, Analysis of phthalate acid esters in environmental water by magnetic graphene solid phase extraction coupled with gas chromatography-mass spectrometry, J. Chromatogr. A, 2014, 1329, 24-29.

15 K. Molaei, H. Bagheri, A. A. Asgharinezhad, H. Ebrahimzadeh and $\mathrm{M}$. Shamsipur, $\mathrm{SiO}_{2}$-coated magnetic graphene oxide modified with polypyrrolepolythiophene: a novel and efficient nanocomposite for solid phase extraction of trace amounts of heavy metals, Talanta, 2017, 67, 607-616.

16 J. M. Chen, J. Zou, J. B. Zeng, X. H. Song, J. J. Ji, Y. R. Wang, J. Ha and X. Chen, Preparation and evaluation of graphenecoated solid-phase microextraction fiber, Anal. Chim. Acta, 2010, 678, 44-49.

17 X. Zhang, S. Chen, Q. Han and M. Ding, Preparation and retention mechanism study of graphene and graphene oxide bonded silica microspheres as stationary phases for high performance liquid chromatography, J. Chromatogr. A, 2013, 1307, 135-143.

18 X. L. Liu, X. Liu, X. Liu, L. P. Guo, L. Yang and S. T. Wang, Graphene oxide and reduced graphene oxide as novel stationary phases via electrostatic assembly for opentubular capillary electrochromatography, Electrophoresis, 2013, 34, 1869-1876.

19 Y. Feng, C. G. Hu, M. L. Qi, R. N. Fu and L. T. Qu, Separation performance of graphene oxide as stationary phase for capillary gas chromatography, Chin. Chem. Lett., 2015, 26, 47-49.

20 J. Fan, M. L. Qi, R. N. Fu and L. T. Qu, Performance of graphene sheets as stationary phase for capillary gas chromatographic separations, J. Chromatogr. A, 2015, 1399, 74-79.

21 Q. S. Qu, C. H. Gu and X. Y. Hu, Capillary coated with graphene and graphene oxide sheets as stationary phase for capillary electrochromatography and capillary liquid chromatography, Anal. Chem., 2012, 84, 8880-8890.

22 X. Liu, X. L. Liu, M. Li, L. P. Guo and L. Yang, Application of graphene as the stationary phase for open-tubular capillary electrochromatography, J. Chromatogr. A, 2013, 1277, 93-97.

23 Q. S. Qu, Y. Q. Shen, C. H. Gu, Z. L. Gu, Q. Gu, C. Y. Wang and X. Y. Hu, Capillary column coated with graphene oxide as stationary phase for gas chromatography, Anal. Chim. Acta, 2012, 757, 83-87.

24 P. Dan, Y. Lu, N. J. Kybert, Z. T. Luo and A. T. C. Johnson, Intrinsic response of graphene vapor sensors, Nano Lett., 2009, 9, 1472-1475.

25 A. Bagri, C. Mattevi, M. Acik, Y. J. Chabal, M. Chhowalla and V. B. Shenoy, Structural evolution during the reduction of chemically derived graphene oxide, Nat. Chem., 2010, 2, 581-587.

26 V. Dua, S. P. Surwade, S. Ammu, S. R. Agnihotra, S. Jain, K. E. Roberts, S. Park, R. S. Ruoff and S. K. Manohar, All organic vapor sensor using inkjet-printed reduced graphene oxide, Angew. Chem., Int. Ed., 2010, 49, 2154-2157.

27 H. Yuan, X. S. Du, H. L. Tai, Y. Li, X. L. Zhao, P. F. Guo, X. Yang, Y. J. Su, Z. S. Xiong, Y. D. Jiang and M. Xu, The effect of the channel curve on the performance of micromachined gas chromatography column, Sens. Actuators, B, 2017, 23, 304-310.

$28 \mathrm{H}$. Yuan, X. S. Du, Y. Li, X. L. Zhao and M. Xu, Chromatographic separation of simulants of nerve and blister agents by combining one and two channel columns with different stationary phases, J. Sep. Sci., 2016, 39, 1295-1299.

29 Y. Li, X. S. Du, Y. Wang, H. L. Tai, D. Qiu, Q. H. Lin and Y. D. Jiang, Improvement of column efficiency in MEMSbased gas chromatography column, RSC Adv., 2014, 4, 3742-3747.

30 B. W. Wright, A. Peaden, M. L. Lee and T. J. Stark, Free radical cross-linking in the preparation of non-extractable stationary phases for capillary gas chromatography, $J$. Chromatogr. A, 1982, 248, 17-34.

$31 \mathrm{H}$. Yuan, M. Xu and Q. Z. Huang, Effects of $\mathrm{pH}$ of the precursor sol on structural and optical properties of $\mathrm{Cu}$ doped ZnO thin films, J. Alloys Compd., 2014, 616, 401-407.

32 Y. Zhou, G. Z. Xie, T. Xie, H. Yuan, H. L. Tai, Y. D. Jiang and Z. Chen, A sensitive film structure improvement of reduced graphene oxide based resistive gas sensors, Appl. Phys. Lett., 2014, 105, 033502.

33 C. Mattevi, G. Eda, S. Agnoli, S. Miller, K. A. Mkhoyan, O. Celik, D. Mastrogiovanni, G. Granozzi, E. Garfunkel and M. Chhowalla, Evolution of electrical, chemical, and structural properties of transparent and conducting 
chemically derived graphene thin films, Adv. Funct. Mater., 2009, 19, 2577-2583.

34 J. T. Zhang, J. W. Jiang, H. L. Li and X. S. Zhao, A highperformance asymmetric supercapacitor fabricated with graphene-based electrodes, Energy Environ. Sci., 2011, 4, 4009-4015.

35 S. Stankovich, D. A. Dikin, G. H. B. Dommett, K. M. Kohlhaas, E. J. Zimney, E. A. Stach, R. D. Piner, S. T. Nguyen and R. S. Ruoff, Graphene-based composite materials, Nature, 2006, 442, 282-286.

36 H. Li, J. Wang, H. Liu, C. Yang, H. Xu, X. Li and H. Cui, Solgel preparation of transparent zinc oxide films with highly preferential crystal orientation, Vacuum, 2004, 77, 57-62.
37 S. Maensiri, P. Laokul and V. Promarak, Synthesis and optical properties of nanocrystalline $\mathrm{ZnO}$ powders by a simple method using zinc acetate dihydrate and poly(vinyl pyrrolidone), J. Cryst. Growth, 2006, 289, 102-106.

38 Y. Yang and T. X. Liu, Fabrication and characterization of graphene oxide/zinc oxide nanorods hybrid, Appl. Surf. Sci., 2011, 257, 8950-8954.

39 J. V. Hinshaw and L. S. Ettre, Introduction to open-tubular column gas chromatography, Advansta, Cleveland, 1994.

40 O. C. Compton and S. B. T. Nguyen, Graphene Oxide, Highly reduced graphene oxide, and graphene: versatile building blocks for carbon-based materials, Small, 2010, 6, 711-723. 